1 Ten Ostreobium (Ulvophyceae) strains from Great Barrier Reef corals as a resource for algal

2 endolith biology and genomics

3

4 Marisa M. Pasella ${ }^{1 *}$, Ming-Fen Eileen Lee ${ }^{1}$, Vanessa R. Marcelino ${ }^{2}$, Anusuya Willis ${ }^{3}$, Heroen

$5 \quad$ Verbruggen $^{1}$

6

$7 \quad{ }^{1}$ School of BioSciences, University of Melbourne, Victoria, Australia

$8{ }^{2}$ Centre for Innate Immunity and Infectious Diseases, Hudson Institute of Medical Research,

9 Clayton, VIC, Australia

$10{ }^{3}$ Australian National Algae Culture Collection ANACC, CSIRO National Collections and

11 Marine Infrastructure, Hobart, TAS, Australia

CONTACT

*Corresponding author m.pasella91@gmail.com

\title{
ACKNOWLEDGEMENTS AND FUNDINGS
}

We thank Trevor Bringloe for logistical assistance and Allison Van de Meene of the

University of Melbourne - Biosciences Microscopy Facility for help with microscopy.

20 Authors also thank the Heron Island Research Station staff for the support provided during 
RUNNING TITLE

Ostreobium strains from the Great Barrier Reef

\section{ABSTRACT}

Ostreobium is a genus of siphonous green algae that lives as an endolith in carbonate substrates under extremely limited light conditions and has recently been gaining attention due to its roles in reef carbonate budgets and its association with reef corals. Knowledge about this genus remains fairly limited due to the scarcity of strains available for physiological studies. Here, we report on 10 strains of Ostreobium isolated from coral skeletons from the Great Barrier Reef. Phenotypic diversity showed differences in the gross morphology and in few structures. Phylogenetic analyses of the $t u f \mathrm{~A}$ and $r b c \mathrm{~L}$ put the strains in the context of the lineages identified previously through environmental sequencing. The chloroplast genomes of our strains are all around $80 \mathrm{k}$ bp in length and show that genome structure is highly conserved, with only a few insertions (some containing putative proteincoding genes) differing between the strains. The addition of these strains from the Great Barrier Reef to our toolkit will help develop Ostreobium as a model species for endolithic growth, low-light photosynthesis and coral-algal associations.

\section{KEYWORDS}


Corals are the results of a symbiotic association between animals, algae, and prokaryotes. The zooxanthellae - dinoflagellates in the family Symbiodiniaceae - colonize the coral tissue and provide the coral with its main source of carbohydrates. Over the years, many strains of the Symbiodiniaceae have been isolated, leading to major advances in our understanding of their taxonomy, photosynthesis, cell composition and interactions with the coral host (e.g. Hill et al. 2012, Goyen et al. 2015, LaJeunesse et al. 2018, Tortorelli et al. 2020).

In the calcium carbonate skeleton beneath the coral tissue, a green layer containing the green alga Ostreobium can often be seen. Ostreobium is the second major photosynthetic organism in the coral holobiont, with its biomass often exceeding that of Symbiodiniaceae (Odum \& Odum 1955). Due to its peculiar niche burrowing into limestone substrates, studies of Ostreobium sp. have been few in comparison with Symbiodiniaceae.

Most of the physiological work done on Ostreobium used in situ measurements of pieces of coral skeleton rather than mono-algal culture strains. They highlighted how Ostreobium is shade-adapted and that some of the carbohydrates it produces through photosynthesis are transferred to the coral (Fine \& Loya 2002). A handful of studies have used cultured strains, showing that Ostreobium is able to utilize a light absorption spectrum beyond 700 nm (near-infra-red, Fork \& Larkum 1989, Koehne et al. 1999, Wilhelm \& Jakob 2006).

During the last few years, there has been renewed interest in the genus, including work on its organelle and nuclear genomes (Verbruggen et al. 2017, Repetti et al. 2020, Iha et al. 2021). Although knowledge about the genus is steadily increasing, most of the studies have used a single Ostreobium strain, limiting our ability to generalize conclusions across the entire species complex. This is relevant because even though only a handful of Ostreobium 
species are formally described, environmental sequencing has shown that the Ostreobium clade is old and diverse, originating 500 million years ago and containing at least 80 specieslevel operational taxonomic units (OTUs) (Marcelino \& Verbruggen 2016, Sauvage et al. 2016). Recent work shows that at least some of these Ostreobium OTUs differ in their physiology (Massé et al. 2020, Iha et al. 2021), illustrating the importance of having representative strains of different lineages to understand the breadth of physiological responses across the genus.

Until very recently, only two Ostreobium strains were available from public repositories (SAG strains 6.99 and 7.99). The former was isolated 30 years ago as an epiphyte on a red alga from the Philippines and the latter 20 years ago as an epiphyte of Jania sp. from southern Australia. In a recent paper, 9 closely related strains isolated from the coral Pocillopora acuta from the Aquarium Tropical du Palais de la Porte Dorée (Paris, France; originally from Indonesia) were deposited in the RBCell collection (Biological Resources of Living and Cryopreserved Cells) at the Muséum National d'Histoire Naturelle (MNHN; Paris, France; Massé et al. 2020).

The scarcity of available Ostreobium strains slows progress in understanding the biology of this genus and its interactions with coral and functions in reef decalcification. Here, we present a collection of 10 Ostreobium strains obtained from the skeletons of corals from the Great Barrier Reef, deposited in the Australian National Algal Culture Collection (ANACC, Hobart). Our aims for the paper are to describe the collection, isolation, and culturing procedures of these strains, provide their phylogenetic context and describe and compare their completely sequenced chloroplast genomes. 


\section{METHODS}

Coral fragments were collected with hammer and chisel at different sites and depths at Heron Island (Great Barrier Reef; Fig.1 and Table 1) from 8 colonies of Porites sp., 1 colony of Pavona sp., and one from an unidentified coral. From the green area in the skeleton, fragments of ca. $0.1 \mathrm{~cm}^{3}$ of volume were isolated with pliers and inoculated in $75 \mathrm{~mL}$ culturing flasks at $26^{\circ} \mathrm{C}$ in $\mathrm{f} / 2$ medium (Guillard \& Ryther 1962) but with vitamins provided at $\mathrm{f} / 4$ concentration (Guillard 1975). $\mathrm{pH}$ was kept at $8.1 \pm 0.15$ and salinity at $35 \mathrm{ppm}$. Culturing flasks were transferred in a walk-in incubator under very low illumination: 1-2 $\mu \mathrm{mol} \mathrm{m} \mathrm{s}^{-2}$ of cool white LED light. When Ostreobium filaments started to emerge from the coral skeleton (Fig. 2), they were cut with a sterilized razor blade under an inverted light microscope to collect a single filament at a time. The newly collected filaments were transferred to a 24-well plate with the same medium used for the coral skeleton fragments. Once the filaments covered more than $50 \%$ of an individual well, they were transferred to plates with larger wells until enough biomass was reached for them to be moved to a $200 \mathrm{~mL}$ glass culturing flask. Initially, some cultures were seen to contain diatoms, coccolithophores, prasinophytes, and cyanobacteria that were initially kept at very low levels by subculturing. At that stage, we avoided using antibiotics to allow Ostreobium to retain more of its natural microbiome for an ongoing research project.

Substrains were also processed to obtain unialgal axenic strains at the Australian National Algae Culture Collection (ANACC). The ten Ostreobium substrains were maintained in $50 \mathrm{~mL} \mathrm{f} / 2$ medium in $200 \mathrm{~mL}$ Petri dishes, at $20^{\circ} \mathrm{C}$, under $1 \mu \mathrm{mol} \mathrm{m} \mathrm{m}^{-2} \mathrm{~s}^{-1}$ photons LED light on a 12h:12h light: dark cycle. A small section of each strain was transferred to a $2 \mathrm{~mL}$ Eppendorf tube containing $1 \mathrm{~mL} \mathrm{f} / 2$ containing an antibiotic cocktail (Penicillin $100 \mathrm{mg}$ $\mathrm{L}^{-1}$, Streptomycin $25 \mathrm{mg} \mathrm{L}^{-1}$, Neomycin $25 \mathrm{mg} \mathrm{L}^{-1}$, Kanamycin $75 \mathrm{mg} \mathrm{L}^{-1}$ ) and incubated on a rotary mixer (Ratek, Australia) for $72 \mathrm{~h}$. The biomass was collected with tweezers, washed in 
fresh $\mathrm{f} / 2$ and placed in $4 \mathrm{~mL} \mathrm{f/2}$ in 6 -well plates. Once biomass had increased, subsamples were transferred to $f / 2$ agar plates, and subsequently, emerging filaments were cut off and transferred to 12 -well plates in $2 \mathrm{~mL} \mathrm{f} / 2$. Light microscopy and fluorescence microscopy (Vert.A1 Axio, Zeiss, Germany) with NucBlue DNA stain (Molecular Probes, Life Technologies, USA) was used to determine contamination of each strain, and the above procedure was repeated as needed to ensure cultures with no visible bacteria.

Pictures of each strain were taken with a CANON EOS 600D to characterize the gross morphology. Subsequently, autofluorescence images were taken using LAS X Widefield Systems with DM6000 B upright microscope at 63x magnification at the University of Melbourne- Biosciences Microscope Facility. All pictures of the strains have been deposited in FigShare (https://doi.org/10.6084/m9.figshare.15022026.v1).

For molecular identification and chloroplast genome sequencing, total genomic DNA was extracted following Cremen et al. 2016 and sequenced on the NovaSeq platform (pairedend, 150 bp reads, ca. $20 \mathrm{~Gb}$ per specimen) at GeneWiz (Suzhou, China) from libraries prepared with the Illumina VAHTS Universal DNA kit.

Sequence assemblies were generated de novo with MEGAHIT 1.2.9 (Li et al. 2015),

SPAdes 3.14.1 (Bankevich et al. 2012) and the seed-and-overlap-based techniques

NOVOplasty 4.2 (Dierckxsens et al. 2017) and GetOrganelle 1.7.1a (Jin et al. 2018).

Completeness and circularity of the chloroplast genomes were tested using NOVOplasty 4.2 (Dierckxsens et al. 2017) and manual gap closing was performed by mapping the raw reads against the contigs obtained from the different assemblers in Geneious Prime 2020.1.3 (https://www.geneious.com). GeSeq (Tillich et al. 2017), ARAGORN (Laslett \& Canback 2004) and MFannot (Beck and Lang 2010) were used as annotation tools and their information combined and curated in Geneious. Open reading frames (ORFs) were identified 
following the protocol of (Pasella et al. 2019) and the annotated plastid genome sequences were submitted to GenBank (Table 1).

The phylogenetic affiliation of the strains was inferred using two different chloroplast-encoded molecular markers derived from the genomes: elongation factor $\mathrm{Tu}$ (tuf $\mathrm{A})$ and RuBisCo large subunit $(r b c \mathrm{~L})$. To provide context, we added tufA sequences of 79 operational taxonomic units (OTUs) identified as Ostreobium sp. (Marcelino \& Verbruggen 2016) and 56 tufA of other Bryopsidales lineages as outgroups (Table S1). For $r b c \mathrm{~L}$, we included $81 \mathrm{rbcL}$ identified as Ostreobium sp., including the $r b c \mathrm{~L}$ sequences of Gutner-Hoch \& Fine 2011 and Massé et al. 2020 and 20 rbcL of other Bryopsidales (table S1). For both molecular markers, sequences were aligned using MUSCLE (Edgar 2004) in Geneious Prime 2020.1.3 (https://www.geneious.com) and the phylogenetic tree for tufA was inferred with maximum likelihood using RAxML v8.0.26 (Stamatakis 2014). We used GTR $+\Gamma$ as the model of sequence evolution and branch support was estimated using 100 bootstrap replicates.

\section{RESULTS \& DISCUSSION}

We initially isolated 11 strains of Ostreobium from coral skeleton fragments collected on Heron Island (Australia, GBR). Strain VRM647 died soon after we sequenced it, so ten unialgal strains were deposited in the Australian National Algal Culture Collection (ANACC, CSIRO, Hobart; Table 1). After several months of growth at low illumination, the free-living thalli showed apparent variation, with gross morphology varying from very compact, dark green thalli to relatively diffuse structures with filaments that appear less pigmented despite growing in identical conditions. Strain VRM650 (Fig. 4) presented very dark compact thalli, while strains VRM605, VRM609, VRM623, VRM633, VRM644 and VRM646 shared the 
same diffuse morphology (Fig. 5) while only presented and the remaining strains showed an intermediate filament density.

All strains are composed of undifferentiated cylindrical filaments with a diameter between 8 and $13 \mu \mathrm{m}$. No swellings or cross-walls were observed in any filaments. For strain VRM623, we found constrictions at random intervals as reported previously for Ostreobium constrictum (Lukas 1974). Kobara \& Chihara (1992) reported "sporangia-type organs" in Ostreobium, occurring at the ends of some filaments. We also observed these in all of our strains, and we noticed new filament growth on the glass culture flasks for several strains. While we could not make microscopic observations to confirm whether these filaments grew from spores that were released from the sporangia-like structures, this is the most likely explanation for our observations.

Chloroplasts were not observed to be reticulated as reported by Lukas (1974). Instead, they were large and ovoid and often nearly as wide as the filaments for all the strains (Fig. 3). The chloroplasts appeared to be homogeneously distributed along all the filaments, in contrast to previous reports of free-living cultures of Ostreobium, where the chloroplasts were reported to be small and mainly distributed against the cytoplasmic side of the cell membrane (Massé et al. 2020).

Ostreobium diversity is mostly known from environmental sequencing. Different studies have used either tufA or $r b c \mathrm{~L}$ as a marker gene, and as a consequence, two alternative classifications have been proposed (Gutner-Hoch \& Fine 2011; Marcelino et al. 2016; Sauvage et al. 2016; Massé et al. 2020). Here, we provide phylogenies for the newly established strains of Ostreobium sp using both markers in order to place the strains in both systems. 
belong to lineage 3 in the tufA phylogeny, while the strains from the deeper-water Tenements site are part of lineage 4, except for VRM644, which is in lineage 3. Among the strains in lineage 3, four strains (VRM605, VRM609, VRM627, and VRM633) have identical tufA sequences, indicating that they are conspecific, and VRM623 is very closely related to them (98.5\% similarity). In lineage 4, VRM650 and VRM647 are likely conspecific $(99.2 \%$ similarity). The remaining strains are more distantly related and recovered in different positions in the phylogenetic tree (Fig. 6).

The strains in the $r b c \mathrm{~L}$ tree were annotated with the classification provided by Massé five strains belong to different clades (Table 1; Fig. 1S). Strain VRM644 is most closely related to the P1-clade strains isolated by Massé et al. (2020). the strains except VRM623 (Table S2). The size and the GC content of the newly sequenced Ostreobium chloroplast genomes are similar to those reported previously (Marcelino et al. 2016, Del Campo et al. 2017, Verbruggen et al. 2017). A total of three ribosomal RNAs and 77 chloroplast protein-coding genes were shared by all the newly assembled chloroplast previously reported for this alga (Marcelino et al. 2016; Verbruggen et al. 2017). The large ribosomal protein L19 ( $r p l 19$ ) gene was lost in four closely-related Ostreobium strains in the lineage 3 (Table S2). The ribosomal protein L19 contributes to bridging the two ribosomal subunits in bacteria (Gao et al. 2003) and it is not currently clear whether the loss observed in 
these Ostreobium strains represents a transfer to the nucleus or a genuine loss. The gene has been lost from the plastid genome on several occasions in the evolution of green-type algae (Uthanumallian et al. 2021), but this is the first time that it was shown to be lost from the plastid in the order Bryopsidales.

All the chloroplast genomes appeared to have only slight variations in the genome size, except for the strain VRM644 that presented two fairly large insertions, one being a 1.8 $\mathrm{kb}$ insertion between the $r p l 32$ and $r p s 9$ genes and the second a $1.3 \mathrm{~kb}$ insertion between the $p s b \mathrm{H}$ and $c l p \mathrm{P}$ genes. In each insertion, we found a freestanding (i.e. non-intronic) ORF (orf237 and orf100) but BLAST searches did not identify any similarity with previously annotated protein-coding genes in green algal chloroplast genomes. Similarly, searches of the amino acid sequences in the NCBI conserved domain search did not return any results. So, even though the origins and functions of many ORFs in the plastid genomes of Bryopsidales remains unclear, recent transcriptomic work showed that many of them are expressed (Zou et al. 2021), suggesting that they do serve a function in the cell.

Three others freestanding ORFs were found in other Ostreobium chloroplast genomes, of which two were exclusive to VRM642, inserted between chlB and psaA (orf122 and orf134; table S2), and one was found in all the strains between the genes psbA and chlI. It showed significant sequence similarity to a previously reported freestanding ORF encoding a group II intron RT/maturase in Bryopsidales. The freestanding group II intron RT/maturase in the Ostreobineae is hypothesized to play a key role in promoting slicing of the introns, since this lineage does not encode genes that may facilitate splicing of its group II introns (Cremen et al. 2018).

In all the newly sequenced chloroplast genomes, we found group II introns in rpoB, rpl23, rpl5 and rpoC1, as had previously been reported for other Ostreobium strains 
(Marcelino et al. 2016; Verbruggen et al. 2017). However, the presence of introns in other genes was strain-specific (Table S3). Group II introns were also found in rps4 and $y c f 3$ for strains VRM650, VRM647, VRM646 and VRM642.

fragmentation in the Bryopsidales (Cremen et al. 2018). In the newly sequenced Ostreobium

chloroplast genomes, seven strains showed fragmentation of the gene with a frameshift, and

VRM646 having both an in-frame stop codon and a frameshift. The gene tilS of the strain

VRM642 was the only one not presenting any type of fragmentation, as was already reported

for a previously sequenced Ostreobium chloroplast genome (Del Campo et al. 2016).

\section{CONCLUSION}

The isolation of 10 Ostreobium strains from skeleton fragments of Great Barrier Reef corals offers perspectives for expanding our knowledge of the biology of this genus. Along with previous strains, they are a valuable addition to our toolkit for this emerging algal model for low-light photosynthesis, endolithic biology and coral symbiosis research. Our work provided detailed information about their phylogenetic context using both commonly used genetic markers and illustrates variations in their chloroplast genomes.

\section{REFERENCES}

Bankevich A., Nurk S., Antipov D., Gurevich A.A., Dvorkin M., Kulikov A.S., Lesin V.M.,

Nikolenko S.I., Pham S., Prjibelski A.D., Pyshkin A.V., Sirotkin A.V., Vyahhi N., 
Cremen M.C.M., Huisman J.M., Marcelino V.R. \& Verbruggen H. 2016. Taxonomic revision of Halimeda (Bryopsidales, Chlorophyta) in south-Western Australia. Australian Systematic Botany 29:41-54.

Del Campo J., Pombert J.F., Šlapeta J., Larkum A. \& Keeling P.J. 2017. The “other” coral symbiont: Ostreobium diversity and distribution. ISME J. 11:296-9.

Dierckxsens N., Mardulyn P. \& Smits G. 2017. NOVOPlasty: De novo assembly of organelle genomes from whole genome data. Nucleic Acids Research 45.

Edgar R.C. 2004. MUSCLE: Multiple sequence alignment with high accuracy and high throughput. Nucleic Acids Research 32:1792-7.

Fine M. \& Loya Y. 2002. Endolithic algae: An alternative source of photoassimilates during coral bleaching. Proceedings of the Royal Society B: Biological Sciences 269:1205-10.

Fork D.C. \& Larkum A.W.D. 1989. Light harvesting in the green alga Ostreobium sp., a coral symbiont adapted to extreme shade. Marine Biology 103:381-5.

Gao H., Sengupta J., Valle M., Korostelev A., Eswar N., Stagg S.M., Van Roey P., Agrawal R.K., Harvey S.C., Sali A., Chapman S.C. \& Frank J. 2003. Study of the structural dynamics of the E. coli 70S ribosome using real-space refinement. Cell. 113:789-801.

Goyen S., Pettay D.T., Szabó M., Warner M.E., Evenhuis C., Ralph P.J. \& Suggett D.J. 2015. Functional diversity of photobiological traits within the genus Symbiodinium appears to be governed by the interaction of cell size with cladal designation . New Phytologist 208:370-81.

Guillard R.R.L. 1975. Culture of phytoplankton for feeding marine invertebrates. pp 26- 60. In Smith W.L. \& Chanley M.H (Eds.) Culture of Marine Invertebrate Animals. Plenum Press, New York, USA. In Culture of Marine Invertebrate Animals. pp. 26-60. 
Guillard R.R.L. \& Ryther J.H. 1962. Studies of marine planktonic diatoms: i. Cyclotella nana hustedt, and detonula confervacea (cleve) gran. Canadian Journal of Microbiology 8:229-39.

Gutner-Hoch E. \& Fine M. 2011. Genotypic diversity and distribution of Ostreobium quekettii within scleractinian corals. Coral Reefs. 30:643-50.

Hill R., Larkum A.W.D., Prášil O., Kramer D.M., Szabó M., Kumar V. \& Ralph P.J. 2012. Light-induced dissociation of antenna complexes in the symbionts of scleractinian corals correlates with sensitivity to coral bleaching. Coral Reefs. 31:963-75.

Iha C., Dougan K.E., Varela J.A., Avila V., Jackson C.J., Bogaert K.A., Chen Y., Judd L.M., Wick R., Holt K.E., Pasella M.M., Ricci F., Repetti S.I., Medina M., Marcelino V.R., Chan C. X. \& Verbruggen H. 2021. Genomic adaptations to an endolithic lifestyle in the coral-associated alga Ostreobium. Current Biology 1-10.

Jin J.J., Yu W-B., Yang J.B., Song Y., dePamphilis C.W., Yi T.S. \& Li D.Z. 2020.

GetOrganelle: A fast and versatile toolkit for accurate de novo assembly of organelle genomes. Genome Biology 21:241.

Kobara T. \& Chihara M. 1992. Occurence of the Siponous Green Alga Ostreobium in Japan. Journal of Japanese Botany 67:227-31.

Koehne B., Elli G., Jennings R.C., Wilhelm C. \& Trissl H.W. 1999. Spectroscopic and molecular characterization of a long wavelength absorbing antenna of Ostreobium sp. Biochimica et Biophysica Acta 94:107.

LaJeunesse T.C., Parkinson J.E., Gabrielson P.W., Jeong H.J., Reimer J.D., Voolstra C.R. \& Santos S.R. 2018. Systematic Revision of Symbiodiniaceae Highlights the Antiquity and Diversity of Coral Endosymbionts. Current Biology 28:2570-2580.e6. 
Laslett D. \& Canback B. 2004. ARAGORN, a program to detect tRNA genes and tmRNA genes in nucleotide sequences. Nucleic Acids Research 32:11-6.

Li D., Liu C.M., Luo R., Sadakane K. \& Lam T.W. 2015. MEGAHIT: An ultra-fast singlenode solution for large and complex metagenomics assembly via succinct de Bruijn graph. Bioinformatics. 31:1674-6.

Lukas K.J. 1974. Two Species of the Chlorophyte Genus Ostreobium From Skeletons of Atlantic and Caribbean Reef Corals. Journal of Phycology 10:331-335.

Marcelino V.R. \& Verbruggen H. 2016. Multi-marker metabarcoding of coral skeletons reveals a rich microbiome and diverse evolutionary origins of endolithic algae. Scientific Report. 6:1-9.

Massé A., Domart-Coulon I., Golubic S., Duché D. \& Tribollet A. 2018. Early skeletal colonization of the coral holobiont by the microboring Ulvophyceae Ostreobium sp. Scientific Reports 8:1-11.

Massé A., Tribollet A., Meziane T., Bourguet-Kondracki M.L., Yéprémian C., Sève C., Thiney N., Longeon A., Coute' A. \& Domart-Coulon I. 2020. Functional diversity of microboring Ostreobium algae isolated from corals. Environmental Microbiology 22:4825-46.

Odum H.T.O.\& Odum E.P. 1955. Trophic Structure and Productivity of a Windward Coral Reef Community on Eniwetok Atoll. Ecological Monograph 25:291-320.

Pasella M.M., Verbruggen H., Nelson W.A. \& Díaz-Tapia P. 2019. The phylogenetic position of the morphologically unusual Pleurostichidium falkenbergii (Rhodomelaceae, Rhodophyta) based on plastid phylogenomics. Phycologia. 00:1-7. 
inflated mitochondrial genomes of siphonous green algae reflect processes driving expansion of noncoding DNA and proliferation of introns. PeerJ. 2020:1-23.

Sauvage T., Schmidt W.E., Suda S. \& Fredericq S. 2016. A metabarcoding framework for facilitated survey of endolithic phototrophs with tufA. BMC Ecology 16:1-21.

Stamatakis A. 2014. RAxML version 8: A tool for phylogenetic analysis and post-analysis of large phylogenies. Bioinformatics. 30:1312-3.

Tillich M., Lehwark P., Pellizzer T., Ulbricht-Jones E.S., Fischer A., Bock R. \& Greiner S. 2017. GeSeq - Versatile and accurate annotation of organelle genomes. Nucleic Acids Reearch. 45:W6-11.

Tortorelli G., Belderok R., Davy S.K., McFadden G.I. \& van Oppen, M.J.H. 2020. Host Genotypic Effect on Algal Symbiosis Establishment in the Coral Model, the Anemone

Uthanumallian K., Iha C., Repetti S.I., Chan C.X, Debashish B., Duchene S. \& Verbruggen H. 2021. Tightly constrained genome reduction and relaxation of purifying selection during secondary plastid endosymbiosis. bioRxiv.

Verbruggen H., Marcelino V.R., Guiry M.D., Cremen M.C.M. \& Jackson C.J. 2017. Photosynthesis Research 87:323-9. 
376 Table 1 Metadata for Ostreobium sp. strains isolated in this study. Strain VRM647 died during the COVID-19 lockdown and is not available in

377 ANACC but is included here to provide the collection details and molecular data generated for this study.

\begin{tabular}{|c|c|c|c|c|c|c|c|c|c|}
\hline \multirow{2}{*}{ Strain } & \multirow{2}{*}{$\begin{array}{l}\text { ANACC } \\
\text { accession }\end{array}$} & \multirow{2}{*}{ Geographic Origin } & \multirow{2}{*}{ Depth (m) } & \multirow{2}{*}{ Longitudine } & \multirow{2}{*}{ Latitudine } & \multicolumn{2}{|c|}{ Phylogenetic affiliation } & \multirow{2}{*}{ Coral Taxa } & \multirow{2}{*}{$\begin{array}{l}\text { GenBank } \\
\text { Accession }\end{array}$} \\
\hline & & & & & & $r b c \mathbf{L}$ & tufA & & \\
\hline VRM605 & CS-1379 & Heron Island (Research station beach) & $0.2-3$ & 151.911965 & -23.4435 & CLADE P3/P14 & Lineage 3 & Porites sp. & XXXXX \\
\hline VRM609 & CS-1380 & Heron Island (Research station beach) & $0.2-3$ & 151.911965 & -23.4435 & CLADE P3/P14 & Lineage 3 & Porites sp. & XXXXX \\
\hline VRM623 & CS-1381 & Heron Island (Shark bay) & $0.2-3$ & 151.919524 & -23.4422 & CLADE P3/P14 & Lineage 3 & Porites sp. & XXXXX \\
\hline VRM627 & CS-1382 & Heron Island (Shark bay) & $0.2-3$ & 151.919524 & -23.4422 & CLADE P3/P14 & Lineage 3 & Porites sp. & XXXXX \\
\hline VRM633 & CS-1383 & Heron Island (Shark bay) & $0.2-3$ & 151.919524 & -23.4422 & CLADE P3/P14 & Lineage 3 & Porites sp. & XXXXX \\
\hline VRM638 & CS-1384 & Heron Island (Tenements) & 6 & 151.929604 & -23.4339 & - & - & - & XXXXX \\
\hline VRM642 & CS-1385 & Heron Island (Tenements) & 18 & 151.929604 & -23.4339 & CLADE C & Lineage 4 & Porites sp. & XXXXX \\
\hline VRM644 & CS-1386 & Heron Island (Tenements) & 16 & 151.929604 & -23.4339 & CLADE P1/K & Lineage 3 & Unidentified coral & XXXXX \\
\hline VRM646 & CS-1387 & Heron Island (Tenements) & 18 & 151.929604 & -23.4339 & CLADE P4 & Lineage 4 & Pavona sp. & XXXXX \\
\hline VRM647 & - & Heron Island (Tenements) & 10 & 151.929604 & -23.4339 & CLADE B3 & Lineage 4 & Porites sp. & XXXXX \\
\hline VRM650 & CS-1388 & Heron Island (Tenements) & 16 & 151.929604 & -23.4339 & CLADE B3 & Lineage 4 & Porites sp. & XXXXX \\
\hline
\end{tabular}


FIGURES LEGENDS

\section{Figure 1-5.}

1.Sampling locations of the corals in Heron Island 2. Longitudinal section of a massive coral

1.

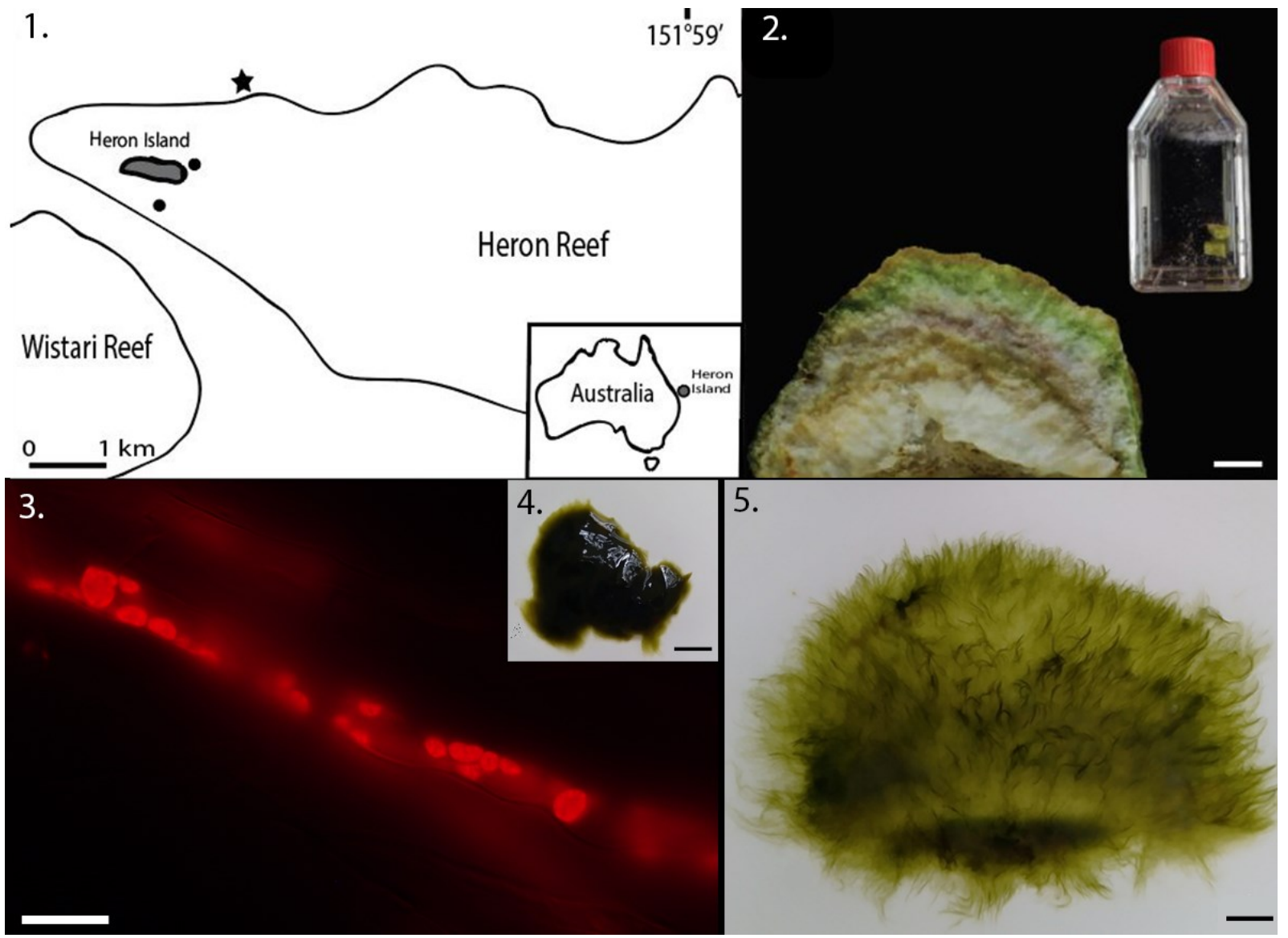


bioRxiv preprint doi: https://doi.org/10.1101/2021.08 16 453452; this version posted August 17, 2021. The copyright holder for this preprint (which was not certified by peer review) is the author/funder, who has granted bioRxiv a license to display the preprint in perpetuity. It is made available under aCC-BY-NC-ND 4.0 International license.

Figure 6

392 Maximum likelihood tree of Ostreobium lineages based on tufA sequences. New strains isolated in this work are

393 indicated in bold. Lineage numbering follows Marcelino et al. (2016). Bootstrap values above 70 are shown.

394 The GenBank accession numbers are listed in Table 1 and Table S1. Note that the phylogeny also includes

395 VRM647, an isolate for which we have molecular data available but that has died.

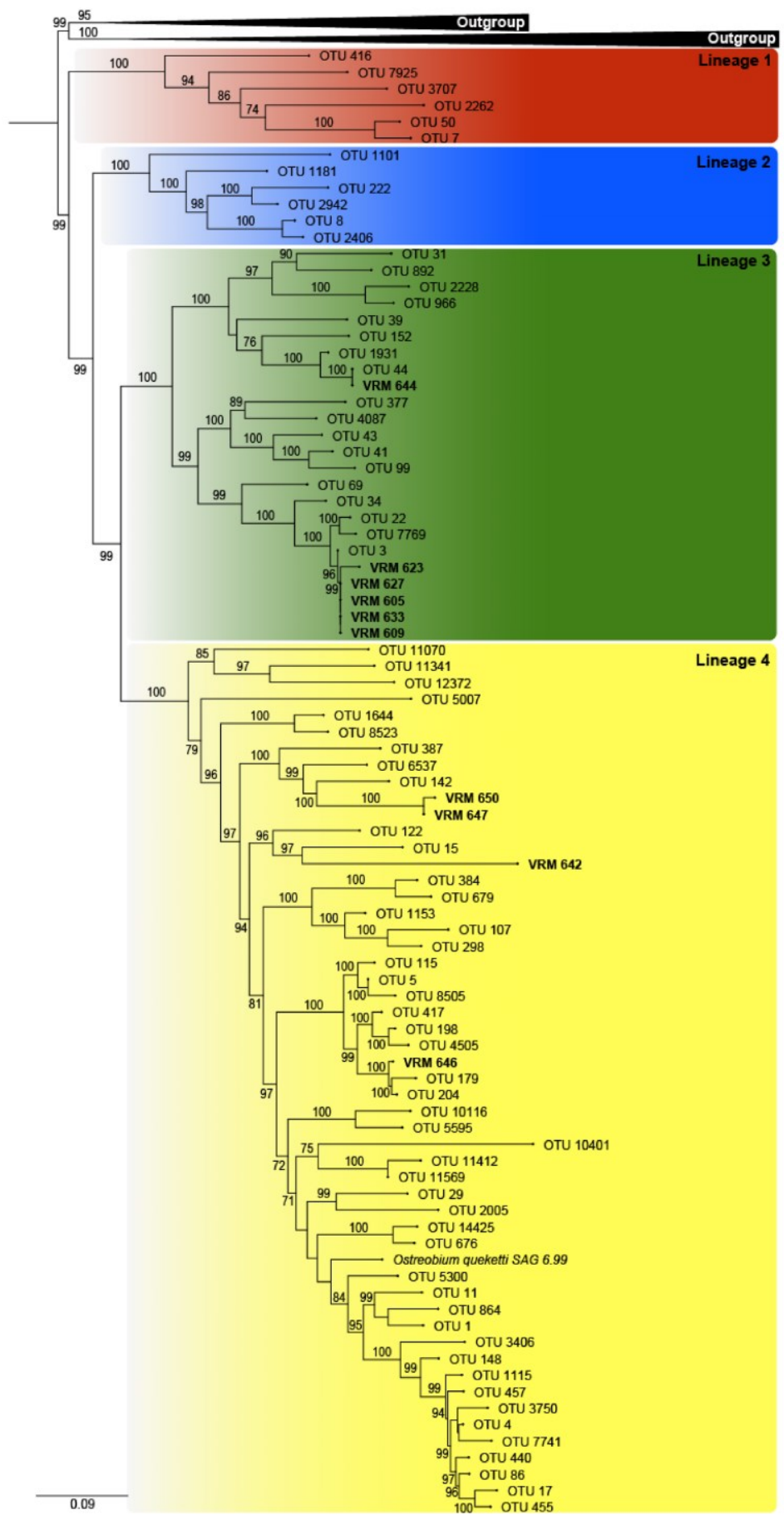




\section{SUPPLEMENTARY MATERIALS}

Figure S1.

400 Maximum likelihood tree of $r c b$ L sequences. Specimens in boldface are the strains isolated in

401 this work. The taxon labels indicate the $r b c$ L clade names from Massé et al. (2018). The colours

402 show how we think the major lineages $3 \& 4$ from the $t u f$ A tree map onto the $r b c \mathrm{~L}$ tree. GenBank

403 accessions of new sequences are listed in Table 1 and Table S1. Only bootstrap values above

40470 are shown.

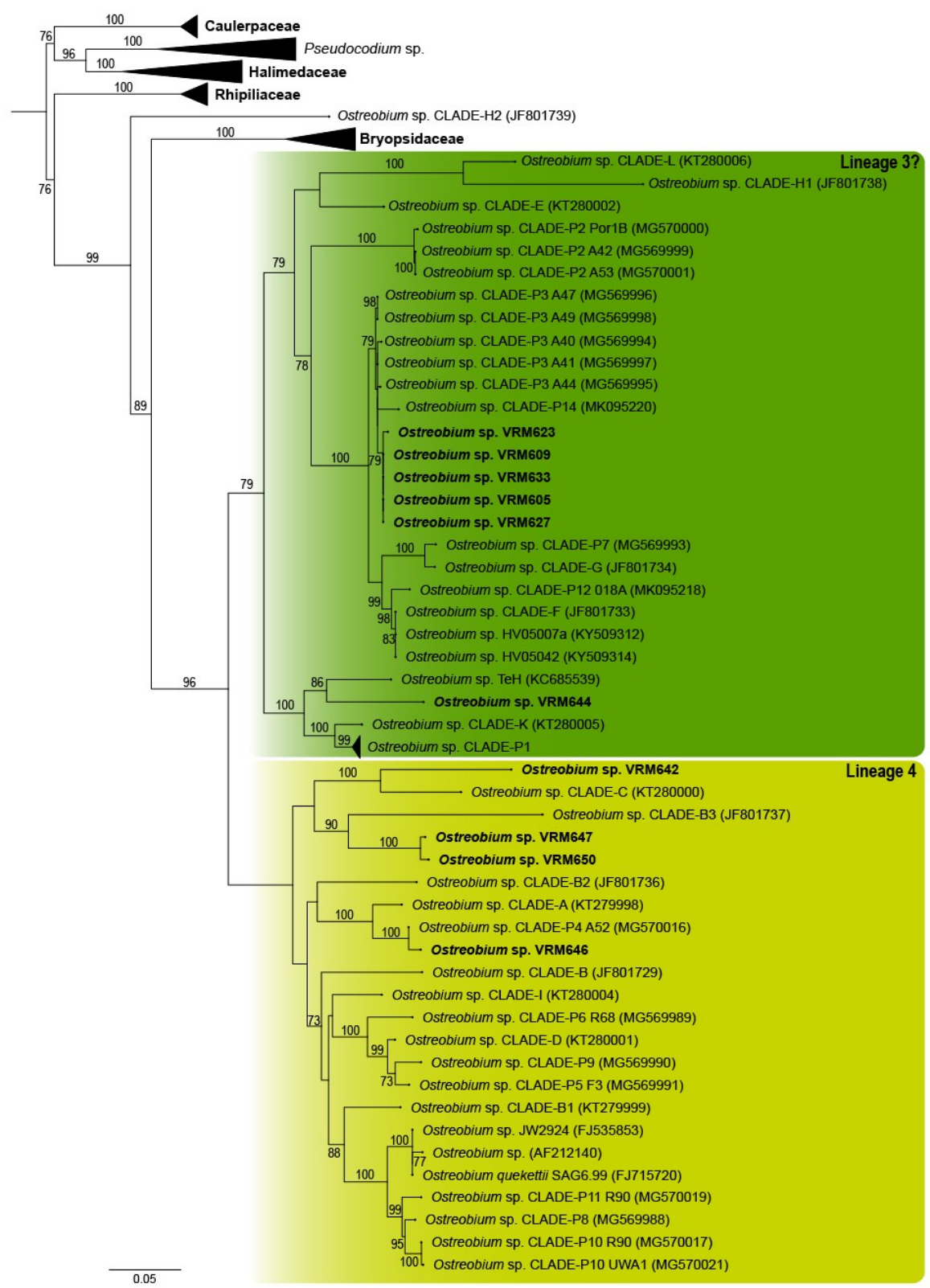


Table S1.

407 Reference sequences Genbank accession number used to reconstruct the green algae tufA

408 phylogeny and outgroup of the rbcL phylogeny.

409

410 Table S2.

411 Features of the chloroplast genome in the Ostreobium strains.

412

413 Table S3.

414 Comparison of the Group II introns distribution in the genes of the Ostreobium sp strains. 